\title{
On the relationship of shock waves to flares and coronal mass ejections
}

\author{
A. Nindos ${ }^{1}$, C. E. Alissandrakis ${ }^{1}$, A. Hillaris ${ }^{2}$, and P. Preka-Papadema ${ }^{2}$ \\ ${ }^{1}$ Section of Astrogeophysics, Physics Department, University of Ioannina, 45110 Ioannina, Greece \\ e-mail: anindos@cc.uoi.gr \\ 2 Section of Astronomy, Astrophysics and Mechanics, Physics Department, University of Athens, 15783 Athens, Greece
}

Received 28 February 2011 / Accepted 5 April 2011

\section{ABSTRACT}

\begin{abstract}
Context. Metric type II bursts are the most direct diagnostic of shock waves in the solar corona.
Aims. There are two main competing views about the origin of coronal shocks: that they originate in either blast waves ignited by the pressure pulse of a flare or piston-driven shocks due to coronal mass ejections (CMEs). We studied three well-observed type II bursts in an attempt to place tighter constraints on their origins.

Methods. The type II bursts were observed by the ARTEMIS radio spectrograph and imaged by the Nançay Radioheliograph (NRH) at least at two frequencies. To take advantage of projection effects, we selected events that occurred away from disk center.

Results. In all events, both flares and CMEs were observed. In the first event, the speed of the shock was about $4200 \mathrm{~km} \mathrm{~s}^{-1}$, while the speed of the CME was about $850 \mathrm{~km} \mathrm{~s}^{-1}$. This discrepancy ruled out the CME as the primary shock driver. The CME may have played a role in the ignition of another shock that occurred just after the high speed one. A CME driver was excluded from the second event as well because the CMEs that appeared in the coronagraph data were not synchronized with the type II burst. In the third event, the kinematics of the CME which was determined by combining EUV and white light data was broadly consistent with the kinematics of the type II burst, and, therefore, the shock was probably CME-driven.

Conclusions. Our study demonstrates the diversity of conditions that may lead to the generation of coronal shocks.
\end{abstract}

Key words. Sun: radio radiation - shock waves - Sun: corona - Sun: flares - Sun: coronal mass ejections (CMEs)

\section{Introduction}

The dynamic spectra of type II radio bursts appear as slowly drifting bands, from high to low frequencies. Most type II bursts appear typically below $150 \mathrm{MHz}$ (Maxwell \& Thompson 1962; Mann et al. 1996), but occasionally the starting frequency may be as high as $500 \mathrm{MHz}$ (Pohjolainen et al. 2008). The frequency drift rates of type II bursts are on the order of $0.3 \mathrm{MHz} / \mathrm{s}$ and are found to increase with increasing starting frequency in the meter wavelength range (e.g. Mann et al. 1995, 1996). Under the assumption that the exciter propagates along the gradient of a typical coronal density model, the drift rate yields speeds of $500-1000 \mathrm{~km} \mathrm{~s}^{-1}$, whereas the Alfvén speed in the low corona is thought to be typically several hundred kilometers per second; this led to the idea that metric type II bursts are generated by coronal MHD shocks travelling at several times the local Alfvén speed. The radio emission is generated at the local plasma frequency and/or its second harmonic; it is produced by either energetic electrons accelerated at the shock front or plasma turbulence excited by the shock. Detailed discussions of the properties of type II bursts are given by Nelson \& Melrose (1985), Vršnak \& Cliver (2008), Nindos et al. (2008), and Pick \& Vilmer (2008).

While there is a concensus that coronal mass ejections (CMEs) are the drivers of interplanetary shocks (e.g. Gopalswamy 2006), the origin of coronal shock waves is not completely understood. Two possible physical interpretations have been proposed: a blast wave ignited by the pressure pulse of a flare, or, alternatively, a piston-driven shock due to a CME.
Small-scale ejecta are also considered as a possible material driver of the shock (e.g. Gopalswamy et al. 1998; Klein et al. 1999; Klassen et al. 2003; Dauphin et al. 2006). These structures act as temporary pistons, generating an initially driven shock. After the expansion stops, the disturbance travels as a blast shock. Statistical studies reveal that most, if not all, metric type II bursts occur during events where both flares and CMEs are observed. The appearance of metric type II bursts without CMEs is exceptionally rare. On the other hand, whenever a metric type II burst without a flare is observed, the flare probably occurred behind the limb.

There have been many studies of the possible association of type II bursts with either flares or CMEs (e.g. Classen \& Aurass 2002; Shanmugaraju et al. 2006; Hudson \& Warmuth 2004; Cho et al. 2005, 2007; Pohjolainen \& Lehtinen 2006; Reiner et al. 2007; Vršnak et al. 2006; Dauphin et al. 2006; Liu et al. 2009). Most type II bursts that lack a clear CME association occur with flares within $30^{\circ}$ of central meridian where CMEs are difficult to observe (e.g. Classen \& Aurass 2002). The traditional argument in favor of a flare origin of coronal shocks is based on the back extrapolation of the type II lanes, which usually corresponds to the interval between the onset and the peak energy release of the flare (Harvey 1965; Klassen et al. 1999; Vršnak 2001a). However, the flare impulsive phase is often closely associated with the acceleration phase of the flare-related CME (e.g. Zhang et al. 2001, 2004; Temmer et al. 2008).

Few comparisons between type II burst imaging and whitelight CMEs have been reported because the heights from 
Table 1. Parameters of flares and CMEs.

\begin{tabular}{lcccc}
\hline \hline Event & Location & $\begin{array}{c}\text { GOES } \\
\text { class }\end{array}$ & $\begin{array}{c}\text { Soft X-rays } \\
\text { Start Peak }\end{array}$ & $\begin{array}{c}\text { CME speed } \\
\left(\mathrm{km} \mathrm{s}^{-1}\right)\end{array}$ \\
\hline March 2, 2000 & S19W60 & M6.5 & $13: 3513: 43$ & 850 \\
March 7, 2000 & S13W60 & C6.3 & $14: 2114: 30$ & - \\
May 2, 2000 & N22W68 & M2.8 & $14: 4214: 51$ & 1278 \\
\hline
\end{tabular}

Table 2. Parameters of type II bursts.

\begin{tabular}{lccccc}
\hline \hline Event & $\begin{array}{c}\text { Frequency range } \\
(\mathrm{MHz})\end{array}$ & $\begin{array}{c}\text { Start-end } \\
\text { time (UT) }\end{array}$ & $\begin{array}{c}\text { Drift rate } \\
\left(\frac{1}{f} \frac{\mathrm{d} f}{\mathrm{~d} t}, \mathrm{~s}^{-1}\right)\end{array}$ & $\begin{array}{c}\text { Type II onset } \\
(\mathrm{UT})\end{array}$ & $\begin{array}{c}\text { NRH beam } \\
\left({ }^{\prime \prime}, 236 \mathrm{MHz}\right)\end{array}$ \\
\hline March 2, 2000 & $154-274$ & $13: 41: 01-13: 41: 27$ & -0.020 & $13: 40: 03$ & $155 \times 254$ \\
March 2, 2000 $^{b}$ & $7.8-70 X$ & $13: 41: 40-13: 57: 00$ & $-0.001^{c}$ & $13: 20: 25-13: 25: 27$ & - \\
March 7, 2000 & $119-249$ & $14: 25: 50-14: 26: 53$ & $-0.008--0.010$ & $14: 24: 12-14: 24: 50$ & $286 \times 148$ \\
May 2, 2000 & $127-340$ & $14: 48: 04-14: 52: 53$ & -0.005 & $14: 45: 10$ & $208 \times 135$ \\
\hline
\end{tabular}

Notes. ${ }^{(a)}$ High-frequency type II burst. ${ }^{(b)}$ Low-frequency type II burst. ${ }^{(c)}$ F2a lane.

which the metric emission originates are usually occulted in space-borne coronagraphs. A direct comparison is also difficult because electron acceleration at shocks may be restricted to quasi-perpendicular regions such that some difference between spatially restricted sources of type II emission and the CME is expected. When simultaneous images are available, the type II burst usually appears lower than the CME front (e.g. Gary et al. 1984; Klein et al. 1999), even though in some cases the shock source is located at or near the CME front (e.g. Maia et al. 2000).

The strongest support of the CME-driven-shock scenario is probably that provided by observations of broadening and intensity changes in the UV emission lines in front of CMEs, which have been attributed to shocks temporally associated with type II bursts (Raymond et al. 2000; Mancuso et al. 2002; Ciaravella et al. 2005). In addition, though less direct, support is provided by the scaling of CME kinetic energy with the frequency range of type II emission and the high association of EUV waves with CMEs. Gopalswamy (2006) and Gopalswamy et al. $(2005,2009)$ suggested that CMEs are the drivers of shocks producing metric type II emission. They pointed out that an Alfvén speed profile with a local maximum in the corona could explain the discontinuity between type II bursts at meter and longer wavelengths, even if they were generated by the same CME. However, the analysis by Cane \& Erickson (2005) challenges the argument that coronal and interplanetary type II bursts are related to the same driver.

The most compelling support of a flare interpretation of type II bursts was provided by both White et al. (2007) and Magdalenić et al. (2008, 2010). White et al. (2007) presented a type II burst that was associated with a long-duration CME-less X3.6-class flare. Magdalenić et al. (2008, 2010) considered a limb event and using radio and EUV images showed that the impulsive CME acceleration in their event lagged the type II burst that was synchronized with the associated flare.

Statistical studies (and in some cases studies of individual events) often do not yield unambiguous conclusions about the origin of type II bursts. The aim of our article is to study the origin of three well-observed type II bursts. We selected events that occurred away from the disk center (thus taking advantage of projection effects) and for which, in addition to the spectral radio data, positional information about the shock radio sources were available from radioheliograph data. Our data are presented in Sect. 2. The three events are described in Sect. 3. Our conclusions are summarized in Sect. 4.

\section{Observations and data reduction}

We selected three events (see Table 1) that contained type II bursts (see Table 2) with the following criteria: (1) the events were observed by the ARTEMIS radio spectrograph and included in the catalog of type II events presented by Caroubalos et al. (2004); (2) the type II emission was imaged by the Nançay Radioheliograph (NRH) at least at two frequencies. The positional information helped us to remove (at least partially) the ambiguity associated with the determination of the shock heights using the type II drift rates and coronal density models; (3) the events occurred at longitudes equal to or larger than $60^{\circ}$ to take advantage of projection effects. Away from disk center, the determination of the kinematics of both radio sources and CMEs becomes more accurate, and CMEs are also easier to observe.

The ARTEMIS solar radio spectrograph is located at Thermopylae, Greece (Caroubalos et al. 2001). At the times of the events analyzed in this article, the instrument covered the frequency range from 110 to $687 \mathrm{MHz}$, using two receivers operating in parallel. In our study, we used data from the global spectral analyzer (ASG), which is a sweep frequency receiver that covered the full frequency band with a time resolution of 10 samples s$^{-1}$. The NRH (Kerdraon \& Delouis 1997) is located $200 \mathrm{~km}$ south of Paris. At the times of our observations, it consisted of 44 antennas, spread over two arms (east-west and north-south) with respective lengths of $3200 \mathrm{~m}$ and $2440 \mathrm{~m}$. It provided images of the full solar disk at five frequencies $(164,236,327,410$, and $432 \mathrm{MHz}$ ) with sub-second time resolution. For the observations reported here, the resolution of the images at $236 \mathrm{MHz}$ is given in Table 2. The extension of the type II burst emission to lower frequencies than the ones covered by ARTEMIS was studied by using data from the Nançay Decametric Array (DAM; 20-70 MHz) and the WIND/WAVES RAD 2 receiver (1.07513.825 MHz).

In addition to the radio data we used EUV observations from the Extreme Incidence Telescope (EIT) at $195 \AA$ and white-light coronagraph data from Large Angle Spectroscopic COronagraph (LASCO) C2 and C3 coronagraphs. For the overall temporal evolution of the flares, we used GOES X-ray total flux measurements. 
A. Nindos et al.: Shock waves, flares, and CMEs

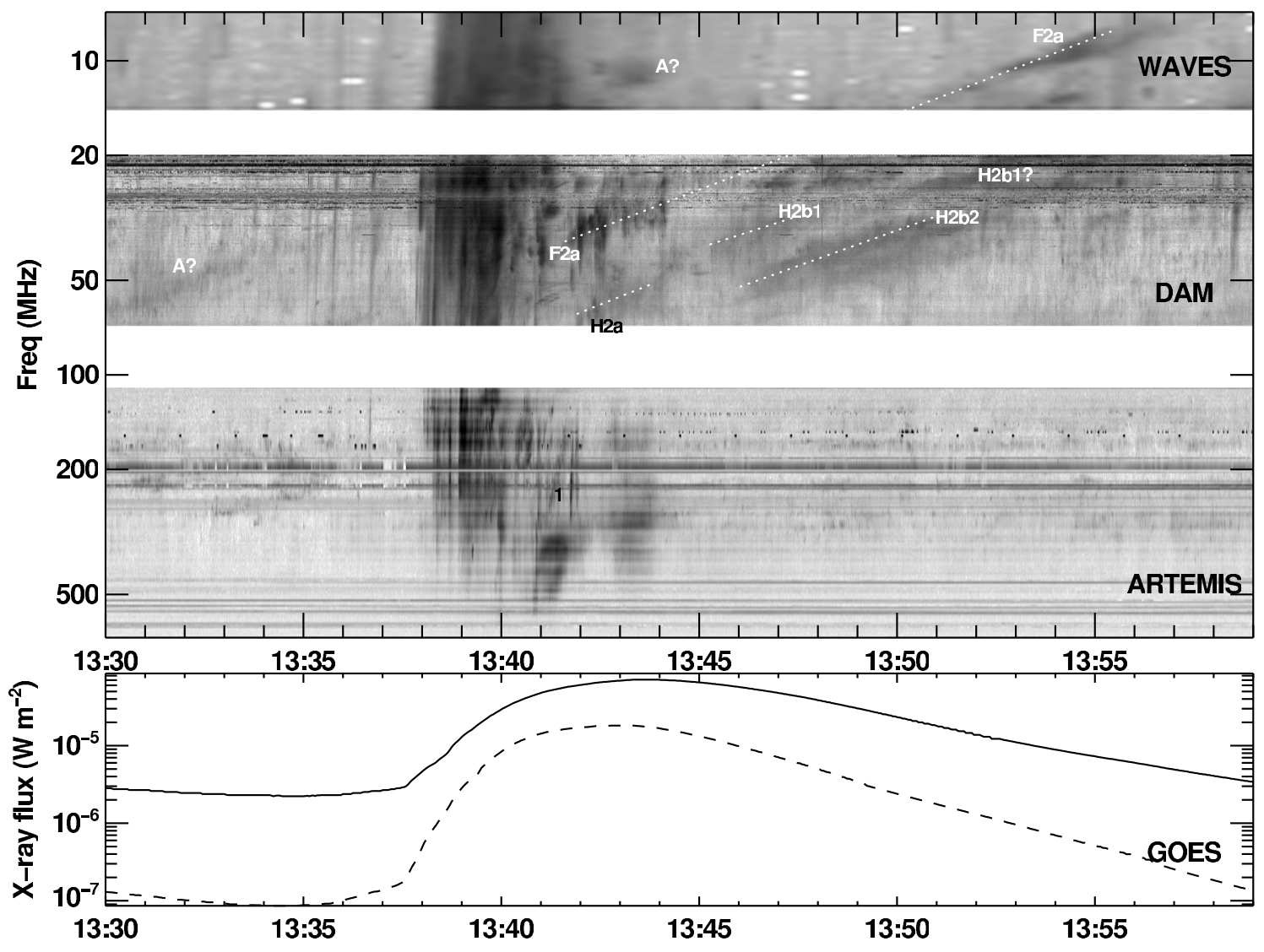

Fig. 1. Upper panel: composite dynamic spectrum of the 2000 March 2 event observed between 687 to 7 MHz. From top to bottom, the Wind/WAVES (7-13.825 MHz), Nançay DAM (20-70 MHz), and ARTEMIS data (110-687 MHz) are presented. Lower panel: time profile

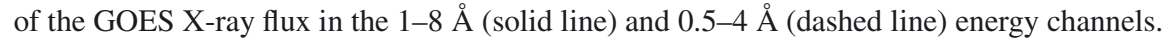

\section{Results}

\subsection{The 2000 March 2 event}

The event of 2000 March 2 was related to a GOES M6.5-class flare that occurred at $19^{\circ} \mathrm{S} 60^{\circ} \mathrm{W}$ (NOAA AR8882). A CME was also observed in the LASCO images. The flare started at 13:35 UT and attained its maximum at 13:43 UT (see Table 1). Before the M6.5-class flare, a C5.5-class flare occurred between 13:06 and 13:26 UT in the same active region. The C5.5-class flare peaked at 13:15 UT. The NRH 10-s resolution data did not show any emission associated with the active region prior to the event. This may be due to its insufficient dynamic range because at the same time a strong compact source close to disk center was present.

Spectral radio observations are presented in the composite dynamic spectrum of Fig.1, which was assembled by combining data from ARTEMIS (687-110 MHz), the Nançay Decametric Array (DAM, 20-70 MHz), and the WIND/WAVES RAD 2 receiver (7-13.825 MHz; only this frequency range was relevant to our event). The ARTEMIS radio data showed no transient emission during the C5.5-class flare. During the interval 13:3813:40 UT, they showed a group of type III bursts associated with the rise phase of the flare that extends to the decametric frequency range. Enclosed between two later type III bursts, there is a type II burst (marked as " 1 " in Fig. 1) whose emission drifts from $274 \mathrm{MHz}$ to $154 \mathrm{MHz}$ within $26 \mathrm{~s}$ (an enlarged view of the ARTEMIS dynamic spectrum is given in Fig. 2). No evidence of a fundamental-harmonic pair of lanes is available for this type II burst. We conclude that we observe the harmonic emission from the shock because in metric type II bursts it is

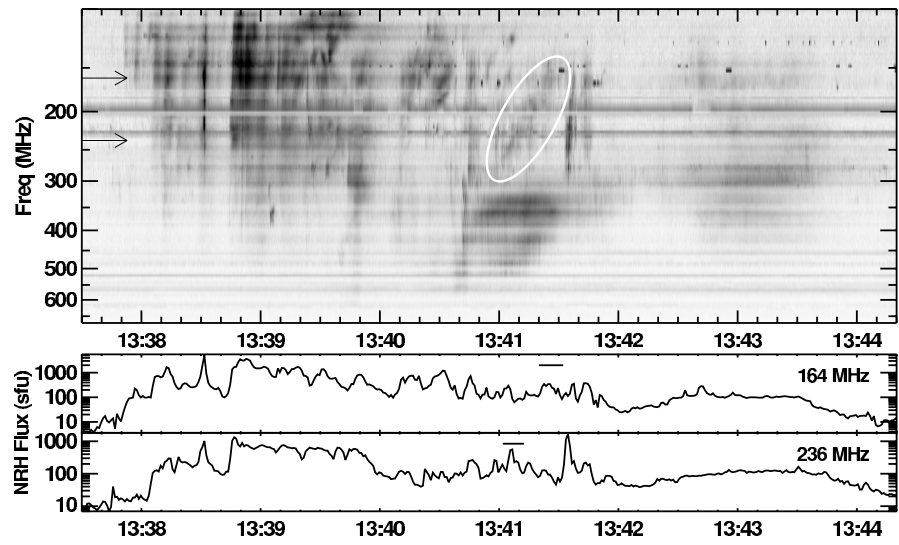

Fig. 2. Upper panel: enlarged view of the dynamic spectrum of the 2000 March 2 event observed by ARTEMIS. The type II burst is surrounded by the white ellipse. The arrows indicate the type II frequencies (236 and $164 \mathrm{MHz}$ ) observed by the NRH. Lower panel: time profiles of the radio flux at 236 and $164 \mathrm{MHz}$ derived from the NRH maps. The horizontal lines mark the passage of the type II burst.

usually stronger than the fundamental (e.g. Vršnak et al. 2001). Continuum emission is observed in the frequency range 296$570 \mathrm{MHz}$ from 13:40:48 UT to 13:42:12 UT and also after the type II burst at frequencies from 130 to $398 \mathrm{MHz}$.

At lower frequencies, the DAM data display two slowly drifting lanes of emission (marked F2a and H2a in Fig. 1), which correspond to the fundamental and second harmonic emissions of another type II burst. Unfornately, owing to the 


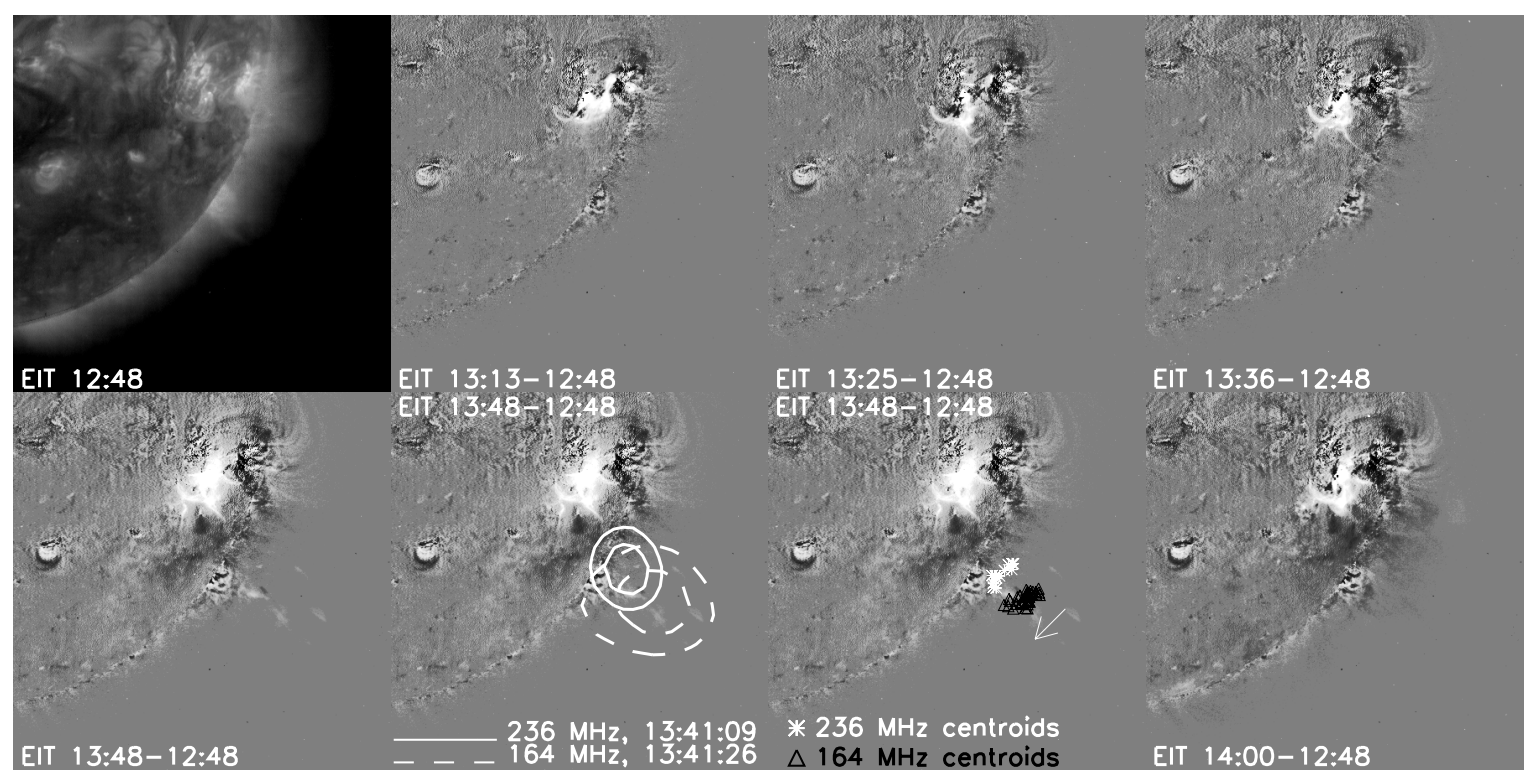

Fig. 3. EIT $195 \AA$ and NRH data of the 2000 March 2 event. The top-left panel shows a pre-event image while the other panels are base difference images (the pre-event image was subtracted from each other image). The panel on the right of the left-bottom panel shows contour plots of the type II source at 236 and $164 \mathrm{MHz}$ obtained by the NRH at 13:41:09 and 13:41:26 UT, respectively (contour levels at 65\% and 85\% of each frequency's maximum). The panel on the left of the right-bottom panel shows the centroids of all NRH sources associated with the type II burst (white stars for $236 \mathrm{MHz}$ and black triangles for $164 \mathrm{MHz}$ ). The arrow denotes the overall direction of motion of the type II radio source at each frequency.

gap between the ARTEMIS and DAM frequencies it is impossible to determine whether the harmonic emission started at frequencies higher than $70 \mathrm{MHz}$. The fundamental emission of the type II burst cannot be clearly distinguished between 13:44 and 13:47 UT in the DAM spectrum, but is probably detected again in the WAVES spectrum from the highest frequency available to its receiver $(13.825 \mathrm{MHz})$ down to $7.8 \mathrm{MHz}$. The harmonic emission of the type II is not visible for about $1 \mathrm{~min}$, but from about 13:45:15 onwards the type II lane marked H2b1 in Fig. 1 starts and within less than one minute so does the $\mathrm{H} 2 \mathrm{~b} 2$ type II lane. These lanes show similar frequecy drifts and therefore they may be considered as "split bands". The nature of the emission patches between the F2a and $\mathrm{H} 2 \mathrm{~b} 1$ lanes is not clear but it is possible that the fundamental band divided into two lanes. If we assume that all the above features are parts of the same shock, its fragmented nature can be interpreted in terms of the varying plasma and magnetic field parameters that suppress radio emission from the shock along a portion of its trajectory. Finally, it is possible that the features marked as "A?" in Fig. 1 (the slowly drifting emission in the DAM spectrum and the blob in the WAVES spectrum) corresponded to an earlier type II burst. It appeared at 13:30 UT, at the time after the end of the C5.5class flare and before the start of the M6.5-class flare.

The low corona signatures of the event are shown in Fig. 3. The EIT data at 13:13 and 13:25 UT show some flare activity in the form of bright loops. These brightenings corresponded to the C5.5 flare while the M6.5 flare was detected in the remaining images of Fig. 3. The difference image at 13:48 UT displays bright small-scale ejecta emanating from the flare site and from small loops located at the limb south of the main flare. In the 13:48 and 14:00 UT images, an EIT wave can be seen propagating south-southeast from the flare site. Its computed speed is $410 \mathrm{~km} \mathrm{~s}^{-1}$, in good agreement with the value reported for the same event by Warmuth et al. (2004). The dimmings observed in the EIT data from 13:25 UT onward indicate that the active region was the source of a CME (images of the CME are given in Fig. 4).

The range of frequencies of the high-frequency type II burst emission permitted its imaging at two NRH frequencies, 236 and $164 \mathrm{MHz}$. Contour plots of two characteristic maps in each frequency are given in Fig. 3. The emission has a compact morphology at both frequencies; the typical dimensions of the sources were $370^{\prime \prime} \times 220^{\prime \prime}$ and $270^{\prime \prime} \times 170^{\prime \prime}$ at 164 and $236 \mathrm{MHz}$, respectively. The NRH sources indicate that the shock was moving almost radially away from the flare site in the general direction of the ejecta. In Fig. 3, the white stars and black triangles denote the positions of the centroids of the NRH sources associated with the type II at 236 and $164 \mathrm{MHz}$, respectively. The arrow denotes that the overall direction of motion of the radio source at each frequency was towards south-southeast which is consistent with the motion of the EIT wave.

The average heights of the centroids of the NRH sources above the photosphere at 236 and $164 \mathrm{MHz}$ were $0.16 R_{\odot}$ and $0.29 R_{\odot}$, respectively. The most extreme heights were $0.13 R_{\odot}$ and $0.19 R_{\odot}$ at $236 \mathrm{MHz}$, and $0.25 R_{\odot}$ and $0.31 R_{\odot}$ at $164 \mathrm{MHz}$. Using the corresponding times, we found that the resulting "average" speed of the shock was $4200 \mathrm{~km} \mathrm{~s}^{-1}$ with extreme values of $3800 \mathrm{~km} \mathrm{~s}^{-1}$ (lower limit) and $4400 \mathrm{~km} \mathrm{~s}^{-1}$ (upper limit). This is probably one of the highest coronal shock speeds reported in the literature (we note, however, that Gergely et al. 1983, reported a shock speed of $4900 \mathrm{~km} \mathrm{~s}^{-1}$ ). We note that all reported shock speeds in this article were not corrected for projection effects to enable us to compare them directly with the associated CME speeds. However, these corrections were small and would increase the shock speeds by less than $10 \%$.

No imaging data were available for the low-frequency type II burst and its speed can be estimated from its frequency drift rate if a coronal density model is provided. The heights of the NRH sources at 236 and $164 \mathrm{MHz}$ were used to select the most appropriate density model. The method we used is similar to 


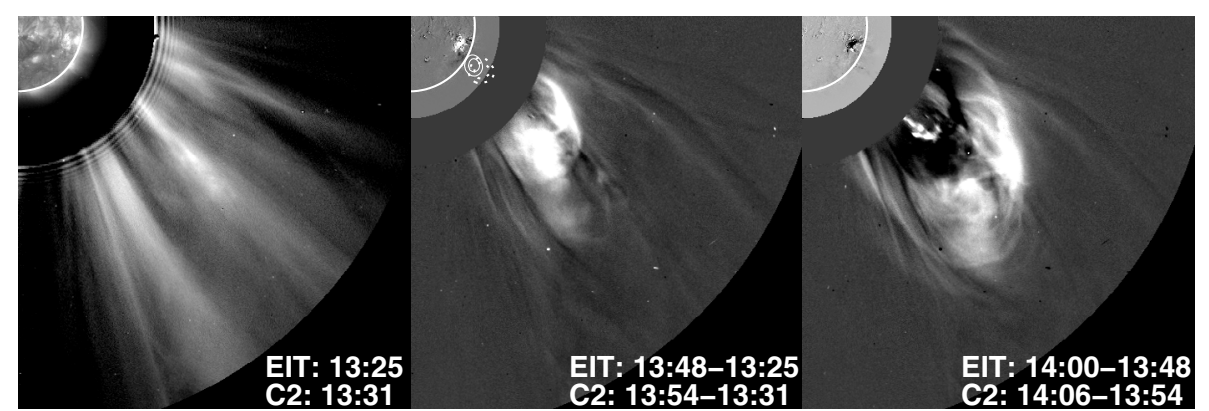

Fig. 4. Pre-event (left panel) and running difference images (middle and right panels) of the CME of the 2000 March 2 event observed by the LASCO C2 coronagraph. In each panel, the LASCO data are combined with the temporally closest EIT data. In the middle panel, the contours are the same as those in Fig. 3. the one presented by Magdalenić et al. (2008, 2010). We obtained the electron densities $N_{e}$ corresponding to the frequencies $f=236 / 2=118 \mathrm{MHz}$ and $f=164 / 2=82 \mathrm{MHz}$, by employing the equation for the plasma frequency $f \approx 9 \sqrt{N_{\mathrm{e}}}$, where $N_{\mathrm{e}}$ is expressed in $\mathrm{cm}^{-3}$ and $f$ in $\mathrm{kHz}$ (since we assumed harmonic emission for the type II burst, the NRH frequencies were converted to the fundamental ones by halving their values). Comparing the radial dependence of the plasma density behavior for different models, we found that the $2.8 \times$ Saito model was the most appropriate model for the ARTEMIS type II burst. When we considered the extreme heights of the NRH sources, the densities of the $2.2 \times$ and $3.2 \times$ Saito models were obtained.

If we assume that the $2.8 \times$ Saito density model is also valid for the heights accessible to the low-frequency type II burst, we find (see also Fig. 5) that the F2a lane corresponded to heights from $0.85 R_{\odot}$ to $1.75 R_{\odot}$ above the photosphere and that its speed was $710 \mathrm{~km} \mathrm{~s}^{-1}$. The $\mathrm{H} 2 \mathrm{a}$ and $\mathrm{H} 2 \mathrm{~b} 2$ lanes corresponded to heights from $0.69 R_{\odot}$ to $0.81 R_{\odot}$, and $0.90 R_{\odot}$ to $1.13 R_{\odot}$, respectively, and their derived speeds were $670 \mathrm{~km} \mathrm{~s}^{-1}$ and $560 \mathrm{~km} \mathrm{~s}^{-1}$, respectively. The $2.2 \times$ and $3.2 \times$ Saito density models yielded heights and speeds that did not vary more than $20 \%$ from the values reported above. However, our method is crude because: (1) we applied the density model derived from a previous type II burst, (2) the CME might have disturbed the densities provided by any coronal model, and (3) radial propagation of the shock was assumed whereas no positional information was available.

The CME (see Fig. 4) was present in the LASCO C2 field of view at 13:54 UT and 14:06 UT, while its first appearance in the C3 field of view occurred at 14:18 UT. The height of the CME was measured at several fixed position angles along its leading front and the results appear in Fig. 5. In the same figure, the diamonds denote the heights reported in the LASCO cat$\operatorname{alog}^{1}$. In most cases, these heights correspond to the outermost CME front propagation. The CME speed in the time interval 13:54-14:06 UT was between 800 and $1050 \mathrm{~km} \mathrm{~s}^{-1}$, while in the time interval 14:06-14:18 UT it was between 780 and $1010 \mathrm{~km} \mathrm{~s}^{-1}$. Using all the $\mathrm{C} 2$ and C3 images in which the CME was present, we found that its average speed was between 750 and $880 \mathrm{~km} \mathrm{~s}^{-1}$ with a speed of $850 \mathrm{~km} \mathrm{~s}^{-1}$ corresponding to the heights along the position angle that intersects the outermost front features. For the computation of the lateral expansion of the CME, we measured the position angles of the intersections of its northern and southern flanks and the solar limb, which were then converted to the along-the-limb distances from the flare site. We found that the lateral speed of the southern and northern flank was 260 and $150 \mathrm{~km} \mathrm{~s}^{-1}$, respectively.

The ability of mass transients to drive shocks depends on the relationship of their speed to the local Alfvén speed. The band split of type II emissions can be attributed to simultaneous emission from the plasma ahead and behind the shock

\footnotetext{
${ }^{1}$ http://cdaw.gsfc.nasa.gov/cme_list
}

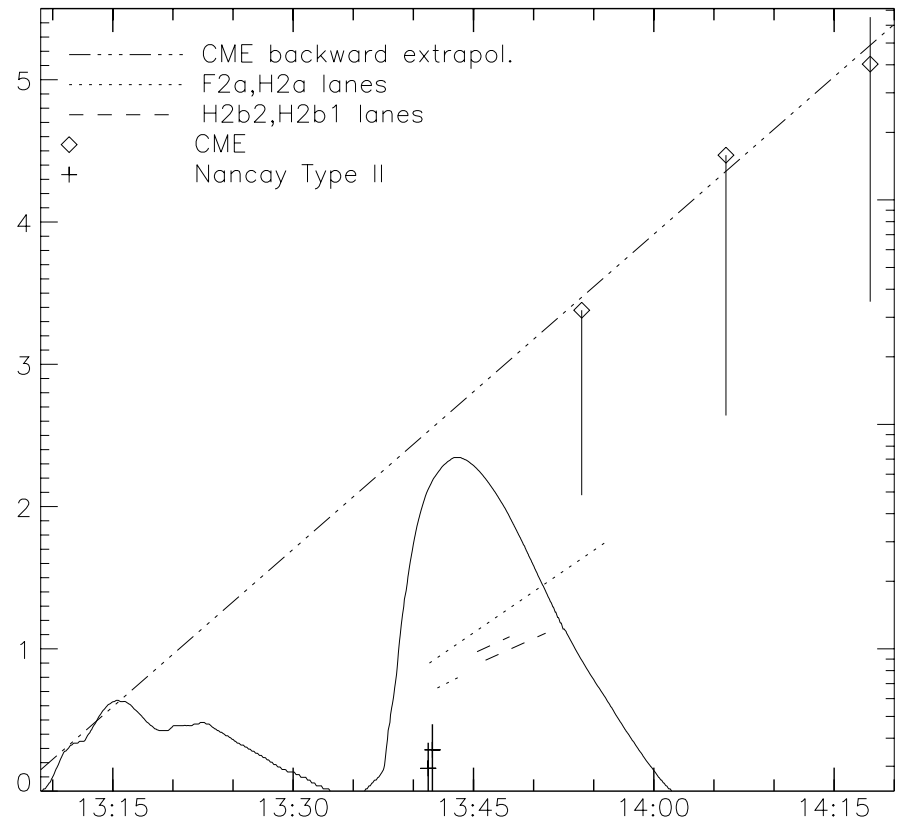

Fig. 5. Height-time profiles of the various components of the 2000 March 2 event (the vertical axis shows heights above the photosphere expressed in solar radii). The diamonds denote the heights of the CME front taken from the CME catalog and the crosses denote the heights of the NRH type II sources. The vertical solid lines associated with the diamonds denote the height difference between the outermost and innermost features of the CME front at a given time. The vertical parts of the crosses denote the sizes of the NRH sources. The dasheddotted curve is the linear fit to the CME heights. The dotted and dashed curves provide the height-time profiles of the $\mathrm{F} 2 \mathrm{a}-\mathrm{H} 2 \mathrm{a}$ and $\mathrm{H} 2 \mathrm{~b} 1-\mathrm{H} 2 \mathrm{~b} 2$ lanes, respectively, of the low frequency type II using the $2.8 \times$ Saito density model. The time profile of the GOES X-ray flux in the $1-8 \AA$ channel is also presented.

(Smerd et al. 1975; Vršnak et al. 2001) and can be used to estimate the Alfvén Mach number and the Alfvén speed. Under the quasi-perpendicular shock approximation and plasma beta $\beta=0$, the Alfvén Mach number $M_{\mathrm{A}}$ is related to the compression $X$ as

$M_{\mathrm{A}}=\sqrt{\frac{X(X+5)}{2(4-X)}}$,

where $X=(1+B D W)^{2}$ and $B D W$ is the average relative frequency separation between the bands (see Priest 1982; Vršnak et al. 2002). We measured the central frequencies $f_{1}$ and $f_{2}$ of the split bands of the harmonic emission of the low frequency type II burst (lanes $\mathrm{H} 2 \mathrm{~b} 1$ and $\mathrm{H} 2 \mathrm{~b} 2$ of Fig. 1) during several time intervals and found that their $B D W=\left(f_{2}-f_{1}\right) / f_{1}$ was 0.31 . From Eq. (1), then we obtained $M_{\mathrm{A}}=1.59$. For a shock speed 
of $v_{\text {shock }}=560 \mathrm{~km} \mathrm{~s}^{-1}$ (see above), the resulting Alfvén speed was $v_{\mathrm{A}}=v_{\text {shock }} / M_{\mathrm{A}}=350 \mathrm{~km} \mathrm{~s}^{-1}$, which is within the values reported in the literature (e.g. Vršnak et al. 2002).

The speed of the CME exceeded the estimated Alfvén speed but the CME is unable to drive the high frequency shock. The primary argument is the discrepancy of a factor of $4-5.25$ between its own speed and the CME speed, if we use the average shock speed of $4200 \mathrm{~km} \mathrm{~s}^{-1}$ and the first measurements of the CME speed. Using the lower and upper limits of the shock speed, we find factors of 3.6-4.75 and 4.2-5.5, respectively, for the discrepancy between the shock speed and the first measurement of the CME speed.

The early evolution of the CME cannot be determined because of the size of the $\mathrm{C} 2$ occulting disk. However, if it exhibited an accelaration phase before entering the LASCO field of view, it is unlikely that it obtained a speed of about $4000 \mathrm{~km} \mathrm{~s}^{-1}$. If that were the case, after the acceleration the CME would have to decelarate from about $4000 \mathrm{~km} \mathrm{~s}^{-1}$ to about $1000 \mathrm{~km} \mathrm{~s}^{-1}$ within $12 \mathrm{~min}$ (this is the time from the end of the high frequency type II burst until the first appearance of the CME in the LASCO images). That would lead to a deceleration of $-4.17 \mathrm{~km} \mathrm{~s}^{-2}$, which is implausible. It is well established that the speed of CMEs does not change much after they obtain their terminal speed by the end of the acceleration phase; a typical CME deceleration is about $-20 \mathrm{~m} \mathrm{~s}^{-2}$ (Gopalswamy et al. 2001), and only in exceptional cases can reach values of about $-150 \mathrm{~m} \mathrm{~s}^{-2}$ (Vršnak 2001b).

The high-frequency type II burst could have been driven by a CME only if (1) the CME was so fast that it could not be observed by the $\mathrm{C} 2$ coronagraph, which would then have insufficient cadence, and (2) its signal was so weak that it could not be imaged by the $\mathrm{C} 3$ coronagraph. The first requirement implies that the CME would need to propagate from the height of the $\mathrm{NRH}$ type II sources to the edge of the $\mathrm{C} 2$ field of view with an average speed of about $4550 \mathrm{~km} \mathrm{~s}^{-1}$, which is consistent with the speed of the shock.

Under the assumptions of radial propagation and a $2.8 \times$ Saito density model, we estimated by extrapolation to the photospheric height the onset time of the type II bursts using their speeds and the heights corresponding to their appearances in the dynamic spectra. The backward extrapolation of the type II burst observed by ARTEMIS and NRH yields its onset time at 13:40:03 UT, which lies within the rise phase of the M6.5-class flare. This may indicate that the shock could be generated by the explosive energy release in the flare (see also Pothitakis et al. 2009). From backward extrapolation of the observed CME, we conclude that it could be related to the earlier C5.5-class flare (see Fig. 5) and this is a second argument against the assumption that the high-frequency type II burst was ignited by that CME.

The situation is less clear concerning the origin of the shock observed by DAM and WAVES. The backward extrapolations (see Fig. 5) of the type II lanes associated with this shock provide onset times of between 13:20:25 and 13:25:27 UT. This time interval lies around the secondary peak of the C5.5-class flare that occurred before the M-class flare (see Fig. 5). We note that the backward extrapolation of the "A?" lane of Fig. 1 yields its onset time at 13:14:30 UT, which lies within the rise phase of the C5.5-class flare.

Figure 5 indicates that the low frequency shock was $0.35 R_{\odot}$ and $1.6 R_{\odot}$ behind the innermost and outermost features, respectively, of the CME front when the CME first appeared in the LASCO C2 field of view. This result is sensitive to the density models used for the calculation of the height-time curves

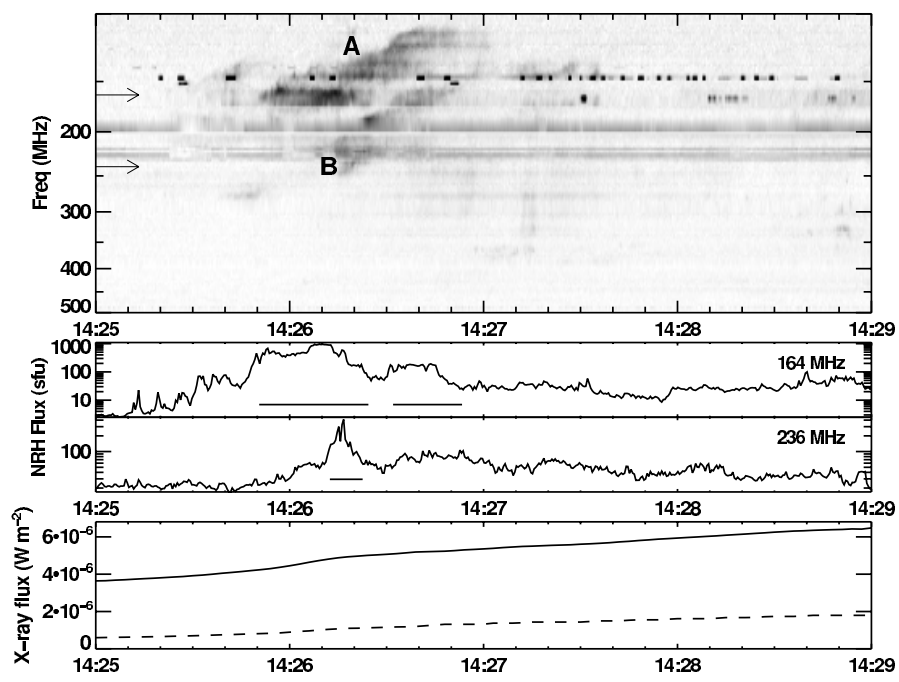

Fig. 6. Same as in Fig. 2 for the 2000 March 7 event with the exception that there is an additional panel where the time profiles of the GOES X-ray flux in the $1-8 \AA$ (solid line) and $0.5-4 \AA$ (dashed line) energy channels are presented.

of the shock. The shock height would match the height of the innermost and outermost features of the CME front if the densities of the $2.8 \times$ Saito model decreased by a factor of 1.5 and 5.4, respectively. Furthermore, Fig. 4 indicates that at 13:54 UT the streamer south of the CME was deflected. This may be evidence of a CME-driven shock running ahead of the CME (e.g. Sheeley et al. 2000; Vourlidas et al. 2003; Ontiveros \& Vourlidas 2009; Liu et al. 2009). This in turn may mean that the shock did not move radially outward because of its refraction into regions of low Alfvén speeds (e.g. Uchida et al. 1973). In this case, the calculation of the speed of the shock using the frequency-drift rate of the type II provides only a lower limit to the true speed of the shock (e.g. Pohjolainen et al. 2007).

\subsection{The 2000 March 7 event}

The type II burst of 2000 March 7 was associated with a GOES C6.3 class flare that occurred in AR NOAA $8891\left(13^{\circ} \mathrm{S} 60^{\circ} \mathrm{W}\right)$. The flare started at 14:21 UT and reached its maximum at 14:30 UT. The dynamic spectrum of the event from ARTEMIS is presented in Fig. 6 (see also Table 2). Before the type II burst, there were no signatures of transient activity in the ARTEMIS spectral records but the WAVES data showed at least four interplanetary type III bursts from about 14:24:15 until about 14:29:30 UT (no DAM observations were available). The NRH 10-s resolution data, however, detected a compact source above the active region at 432 and $164 \mathrm{MHz}$ that was probably associated with a noise storm. According to the catalog of type II bursts compiled by the Astrophysical Institute of Potsdam (AIP), the type II burst of Fig. 6 showed both fundamental and harmonic emission bands; the observing frequencies of ARTEMIS provided data for the harmonic emission band only, which was split into two well-defined lanes marked A and B in Fig. 6. Their relative frequency separation $B D W$ (see Sect. 3.1) varied from 0.30 to 0.33 . After the type II burst and until about 14:50 UT, the ARTEMIS data showed continuum emission from 300-400 to $130 \mathrm{MHz}$. Another type II burst was embedded into the continuum, and displayed two patchy emission lanes that drifted from $315 \mathrm{MHz}$ at 14:30:52 UT to $185 \mathrm{MHz}$ at 14:32:33 UT. 


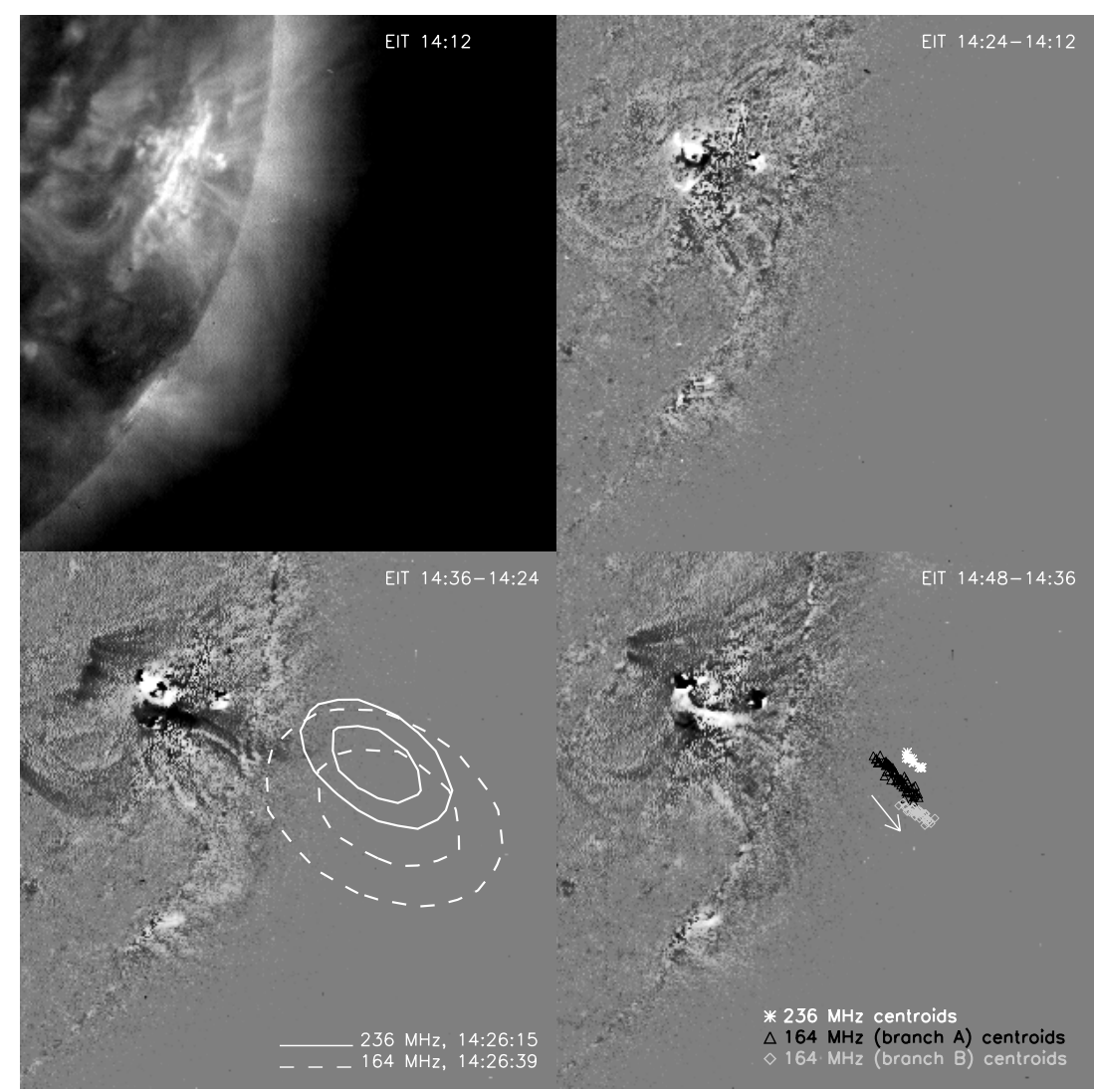

Fig. 7. EIT $195 \AA$ and NRH data of the 2000 March 7 event in the same format as in Fig. 3, with the following exceptions: all EIT images except the one of the topleft panel are running difference images. The bottomleft panel shows contour plots of the type II source at 236 and $164 \mathrm{MHz}$ obtained by the NRH at 14:26:15 and 14:26:39 UT, respectively. The right-bottom panel shows the centroids of all NRH sources associated with the type II burst (white stars for $236 \mathrm{MHz}$, black triangles for $164 \mathrm{MHz}$ sources of branch A, and gray diamonds for $164 \mathrm{MHz}$ sources of branch B).
Using the method described in Sect. 3.1, we found that the backward extrapolation of lanes B and A, yielded the onset time of the type II burst at 14:24:12 and 14:24:50 UT, respectively. We note that the results of this method are practically independent of the coronal density model used to calculate the height and speed of the radio source because a higher density model yields larger heights but also higher speeds and the two effects cancel out (Vršnak et al. 1995).

The EIT data for the event are presented in Fig. 7. The flare appears as rather compact brightenings in the EIT images. At 14:36 UT, there was evidence of some activation of a filamentlike elongated dark feature whose projection on the solar disk ran almost perpendicularly to the bright features of the active region (the activation probably started earlier as indicated by the dark semi-circular feature south of the most prominent brightening of the 14:24 UT difference image). In the two bottom panels of Fig. 7, diffuse loop-like features without appreciable motion appear on the limb south of the flare brightening.

The type II burst was imaged by the NRH at 236 and $164 \mathrm{MHz}$. In Fig. 7, we present contour plots of two characteristic maps in each frequency as well as the positions of the centroids of the NRH sources associated with the type II burst. The emission was unresolved at both frequencies and the typical dimensions of the sources were $150^{\prime \prime} \times 290^{\prime \prime}$ and $260^{\prime \prime} \times 430^{\prime \prime}$ at 236 and $164 \mathrm{MHz}$, respectively. The alignment of the type II source positions deviates from being radial with respect to the associated flare. The non-radial propagation of type II sources has been reported in other cases as well (e.g. Nelson \& Robinson 1975; Klein et al. 1999). Three possible interpretations have been considered for such propagation: refraction of the shock wave into regions of low Alfvén speed (Uchida et al. 1973), preferential acceleration of electrons in restricted regions of the shock front (e.g. Steinolfson 1984), or suitable orientation of the motion of the shock driver as well as the magnetic environment where the type II burst occurs (Aurass et al. 1998; Klein et al. 1999).

The NRH observations imaged both type II bands at $164 \mathrm{MHz}$. The sizes of the sources were similar for both bands and their locations did not overlap; overall, the high frequency band (B in Fig. 6) was further from the solar surface than the low frequency band (A in Fig. 6). In the bottom-right panel of Fig. 7, the arrow indicates the general direction of motion of the radio source at each frequency, which is consistent with the ARTEMIS data indicating that the $164 \mathrm{MHz}$ emission of the low frequency branch appeared earlier than that of the high frequency band. Imaging observations of split-band sources with modern instruments is not frequent; the old Culgoora Radioheliograph data (e.g. Smerd et al. 1975; Nelson \& Robinson 1975) showed the split-band sources to be cospatial on average; when they were not, either band source could be further from the Sun.

The average positions of the centroids of the NRH sources at $236 \mathrm{MHz}$ and those of the high-frequency band at $164 \mathrm{MHz}$ yielded a shock speed of $2300 \mathrm{~km} \mathrm{~s}^{-1}$. If the most extreme positions and times are considered, we obtain a lower limit of $2100 \mathrm{~km} \mathrm{~s}^{-1}$ and an upper limit of $2400 \mathrm{~km} \mathrm{~s}^{-1}$ for the speed of the shock. Given the non-radial propagation of the shock, the use of the drift rate from the spectral data could provide misleading results for the speed of the shock.

In Fig. 8 we present images from the LASCO C2 coronagraph before and after the type II burst. There were two CMEs in the field of view of the instrument (marked as A and B in the figure) but none of them were related to our event, as becomes clear in Fig. 9. Linear fits to their height-time points presented in Fig. 9 yielded onset times at 12:17 and 13:02 UT for CMEs $\mathrm{A}$ and $\mathrm{B}$, respectively. Furthermore, the timing of the dark feature marked as D in Fig. 8 rules out its possible contribution to the shock formation. This feature was probably the remnant of a previous CME, which moved with a speed of 


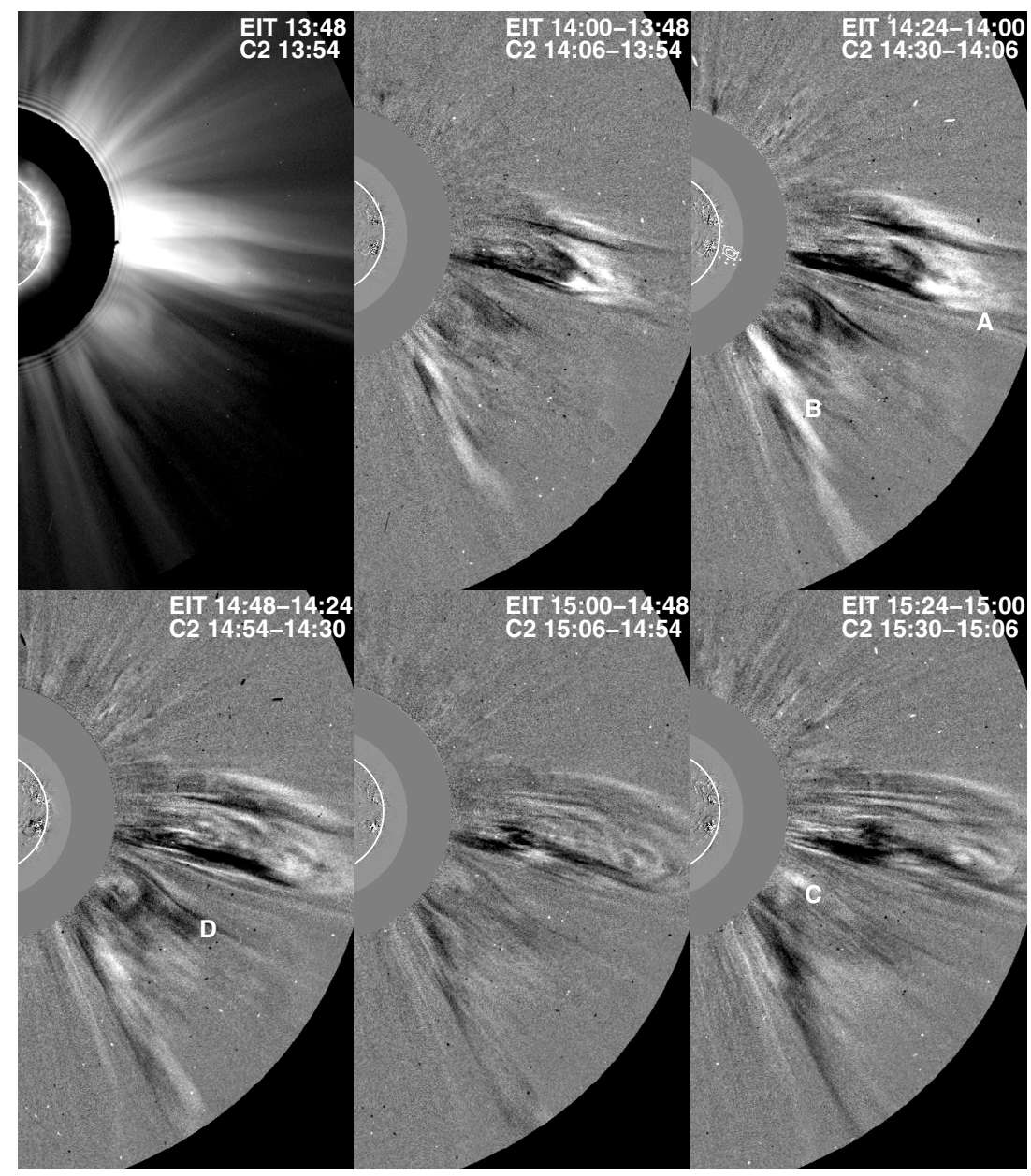

Fig. 8. Pre-event (top-left panel) and running difference images of the 2000 March 7 event observed by the LASCO C 2 coronagraph. In each panel, the LASCO data are combined with the temporally closest EIT data. In the top-right panel, the contours are the same as the ones in Fig. 7.
$230 \mathrm{~km} \mathrm{~s}^{-1}$ between 14:30 and 14:54 UT and then disappeared. In the 15:30 C2 image, there is a narrow bright feature (marked as $\mathrm{C}$ in Fig. 8) in the general direction of the prolongation of the line that connects the flare site and the $236 \mathrm{MHz}$ sources. This feature did not appear in the subsequent C2 image, which was taken at 15:54 UT. Under the assumption that its speed was constant from the height of the $236 \mathrm{MHz}$ source to its height in the $\mathrm{C} 2$ image, its estimated speed was $260 \mathrm{~km} \mathrm{~s}^{-1}$. Our data do not allow us to evaluate its possible contribution to the shock formation in detail. However, it is not likely to be associated with the dark feature whose activation was observed in the EIT images because after its activation it showed no outward motion in the EIT field of view.

\subsection{The 2000 May 2 event}

The type II burst of 2000 May 2 was associated with a GOES $\mathrm{M} 2.8$-class flare that occurred at $22^{\circ} \mathrm{N} 68^{\circ} \mathrm{W}$ (NOAA AR8971) and a CME. The dynamic spectrum of the event from ARTEMIS is presented in Fig. 10. In the ARTEMIS frequency range, the event started with three groups of type III bursts associated with the rise phase of the flare. The WAVES data showed that these bursts extended into the interplanetary medium (no DAM observations were available). The type II burst appeared toward the end of the metric type III activity at about 14:48:04 UT and lasted until 14:52:53. For two minutes, it drifted from $340 \mathrm{MHz}$ to $221 \mathrm{MHz}$. No trace of it could be found in the ARTEMIS spectrum from 14:50:05 to 14:51:24 UT. As mentioned in Sect. 3.1, these interuptions may reflect varying plasma and magnetic field parameters which suppress radio emission from the shock along a portion of its trajectory. The type II appeared again at $170 \mathrm{MHz}$, then drifted to $127 \mathrm{MHz}$ within $90 \mathrm{~s}$. During that time interval, a short continuum was present in the dynamic spectrum close to the type II burst. There was no trace of a fundamentalharmonic pair of lanes for this type II in either the ARTEMIS spectrum or in the available catalogs of type II bursts (the burst appears only in the NOAA catalog as observed from the Bleien station at frequencies from 220 to $280 \mathrm{MHz}$ ). As in the case of the 2000 March 2 ARTEMIS type II burst, we attributed the observed lane to harmonic emission.

The EIT data for the event are presented in Fig. 11. The images taken at 14:36 and 14:48 UT did not show any prominent brightenings, but did show expanding loop-like features. The height of the tip of the expanding loops above the solar surface was $61^{\prime \prime}$ and $91^{\prime \prime}$ at 14:36 and 14:48 UT, respectively, yielding a speed of about $30 \mathrm{~km} \mathrm{~s}^{-1}$. In the 14:36 UT image, there were two dark patches below the activated loops. The dark areas grew in the two subsequent images and probably reflected the dimming of the EUV emission caused by the mass depleted by the CME. The last EIT image was obtained after the end of the type II burst and shows an intense brightening associated with the flare. It also shows large-scale dark loop-like features, which probably represent loops that were carried away by the CME.

Before the event, the NRH 10-s resolution data at $432 \mathrm{MHz}$ detected a compact source at the limb, probably associated with the active region and diffuse emission bridging that source with another source located at the southwest quadrant just above the limb. At $164 \mathrm{MHz}$, the pre-event data showed a compact source 


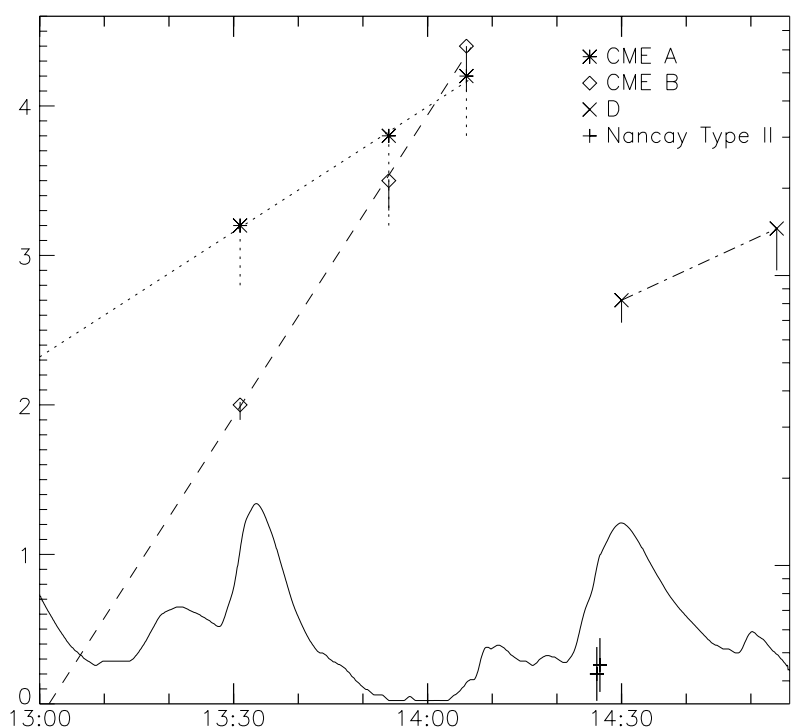

Fig. 9. Height-time profiles of various features of the 2000 March 7 event in the same format as in Fig. 5, with the following exceptions: The stars, diamonds and " $\times$ "s denote the heights of features A, B, and D of Fig. 8. The crosses denote the heights of the NRH type II sources of branch B. The vertical solid and dotted lines associated with the diamonds, stars, and " $x$ "s denote height difference between the outermost and innermost features of their fronts at a given time.

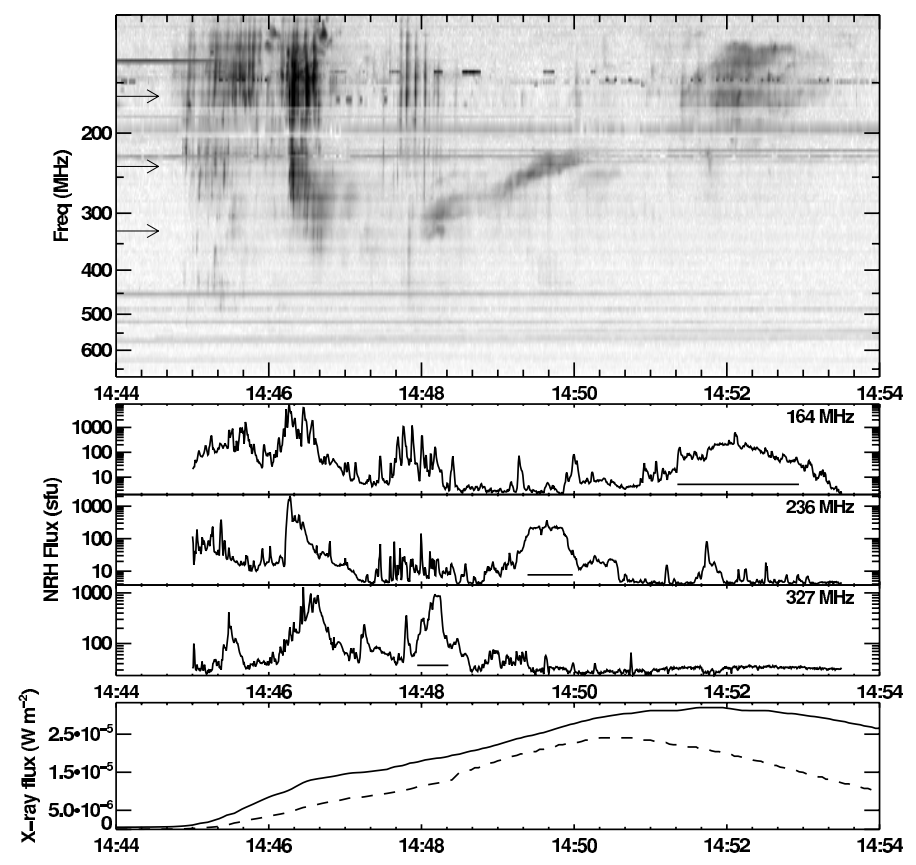

Fig. 10. Same as in Fig. 6 for the 2000 May 2 event.

located about $0.7 R_{\odot}$ beyond the limb in the southwest quadrant of the image.

The type II burst was imaged by the NRH at 327, 236, and $164 \mathrm{MHz}$. Contour plots of one characteristic map at each frequency as well as the positions of the centroids of the NRH sources associated with the type II are given in Fig. 12. The emission of the type II burst consisted of one source at 327 and $164 \mathrm{MHz}$ with typical sizes of $150^{\prime \prime} \times 210^{\prime \prime}$ and $340^{\prime \prime} \times 220^{\prime \prime}$, respectively. The $236 \mathrm{MHz}$ emission consisted of two sources; the stronger was located radially below the $164 \mathrm{MHz}$ sources and the weaker was located radially above the $327 \mathrm{MHz}$ sources. This may indicate that inhomogeneities in the local plasma density and/or magnetic field prevented the formation of radio emission from the entire shock front at 327 and $164 \mathrm{MHz}$. Overall, the alignment of the centroids of the 327 and $236 \mathrm{MHz}$ emissions was non-radial with respect to the flare site, in contrast to the overall alignment between the 236 and $164 \mathrm{MHz}$ centroids. With respect to the expanding loops, the $327 \mathrm{MHz}$ sources appeared at the side of their northernmost features, while the centroids of the 236 and $164 \mathrm{MHz}$ appeared above them.

The shock speeds derived from the average positions of the centroids of the 327 and $236 \mathrm{MHz}$ sources and from those of the centroids of the 236 and $164 \mathrm{MHz}$ sources were 1240 and $1180 \mathrm{~km} \mathrm{~s}^{-1}$, respectively. If the most extreme positions and times are considered, we obtain a lower limit of $1030 \mathrm{~km} \mathrm{~s}^{-1}$ and an upper limit of $1860 \mathrm{~km} \mathrm{~s}^{-1}$ for the former speed and a lower limit of $930 \mathrm{~km} \mathrm{~s}^{-1}$ and an upper limit of $1440 \mathrm{~km} \mathrm{~s}^{-1}$ for the latter speed.

Two CMEs appear in the difference images of Fig. 13; the one associated with our event was the wider one (the north $\mathrm{CME}$ ). The height-time profile of the CME is given in Fig. 14. For the subsequent computations, all heights were measured along the line connecting the disk center with the outermost feature of the CME front at 15:06 UT (in Fig. 14, the resulting CME heights correspond to the diamonds). The position angle of this line measured from Solar west counter-clockwise was $19^{\circ}$. The backward extrapolation of the CME heights using all LASCO images in which the CME appeared (i.e. not only the three images that are represented in Fig. 14) yielded the CME onset time at 14:40 UT. This computation is crude because it does not take into account the possible acceleration of the CME when it was below the $\mathrm{C} 2$ occulting disk. We tried to address this problem by considering the expanding EIT loops at 14:36 and 14:48 UT as signatures of the CME in its infancy. Their projected heights are marked with the squares of Fig. 14. The projected CME heights (including the squares of Fig. 14) were fitted with the function

$$
\begin{aligned}
H(t)= & H\left(t_{1}\right)+\frac{1}{2}\left(v_{\mathrm{f}}+v_{0}\right)\left(t-t_{1}\right) \\
& +\frac{1}{2}\left(v_{\mathrm{f}}-v_{0}\right) \tau \ln \left[\cosh \left(\frac{t-t_{1}}{\tau}\right)\right],
\end{aligned}
$$

where $v_{0}$ and $v_{\mathrm{f}}$ are the initial and final CME speeds, respectively, $t_{1}$ is the time of peak acceleration, and $\tau$ is the timescale of the rise to peak acceleration. This function was used to fit CME height-time profiles by Sheeley et al. (2007) and Patsourakos et al. (2010) and is capable of reproducing a wide range of profiles from almost constant acceleration to impulsive acceleration.

Our best-fit model (the dashed curve of Fig. 14) showed that the CME acceleration peaked at 14:48:15 UT, at a height $H\left(t_{1}\right)=0.2 R_{\odot}$ and lasted for $215 \mathrm{~s}$. It is interesting that the peak acceleration occurred $11 \mathrm{~s}$ after the start of the type II burst. The values of $v_{0}$ and $v_{\mathrm{f}}$ used in our model were $27 \mathrm{~km} \mathrm{~s}^{-1}$ and $1400 \mathrm{~km} \mathrm{~s}^{-1}$, respectively. The maximum value of the acceleration of the CME was $3.2 \mathrm{~km} \mathrm{~s}^{-2}$. At the times when the 236 and $164 \mathrm{MHz}$ shock emission occurred, the model yielded CME speeds of about 1130 and $1380 \mathrm{~km} \mathrm{~s}^{-1}$, respectively. These speeds were derived under the assumption of radial CME propagation whereas the alignment of the centroids of the 327 and $236 \mathrm{MHz}$ sources deviated significantly from the radial direction. The speed of the shock derived from the positions of the 327 and $236 \mathrm{MHz}$ sources had a component along the assumed CME direction of propagation of about $740 \mathrm{~km} \mathrm{~s}^{-1}$. Therefore, the kinematics of the CME and the shock were broadly consistent. 


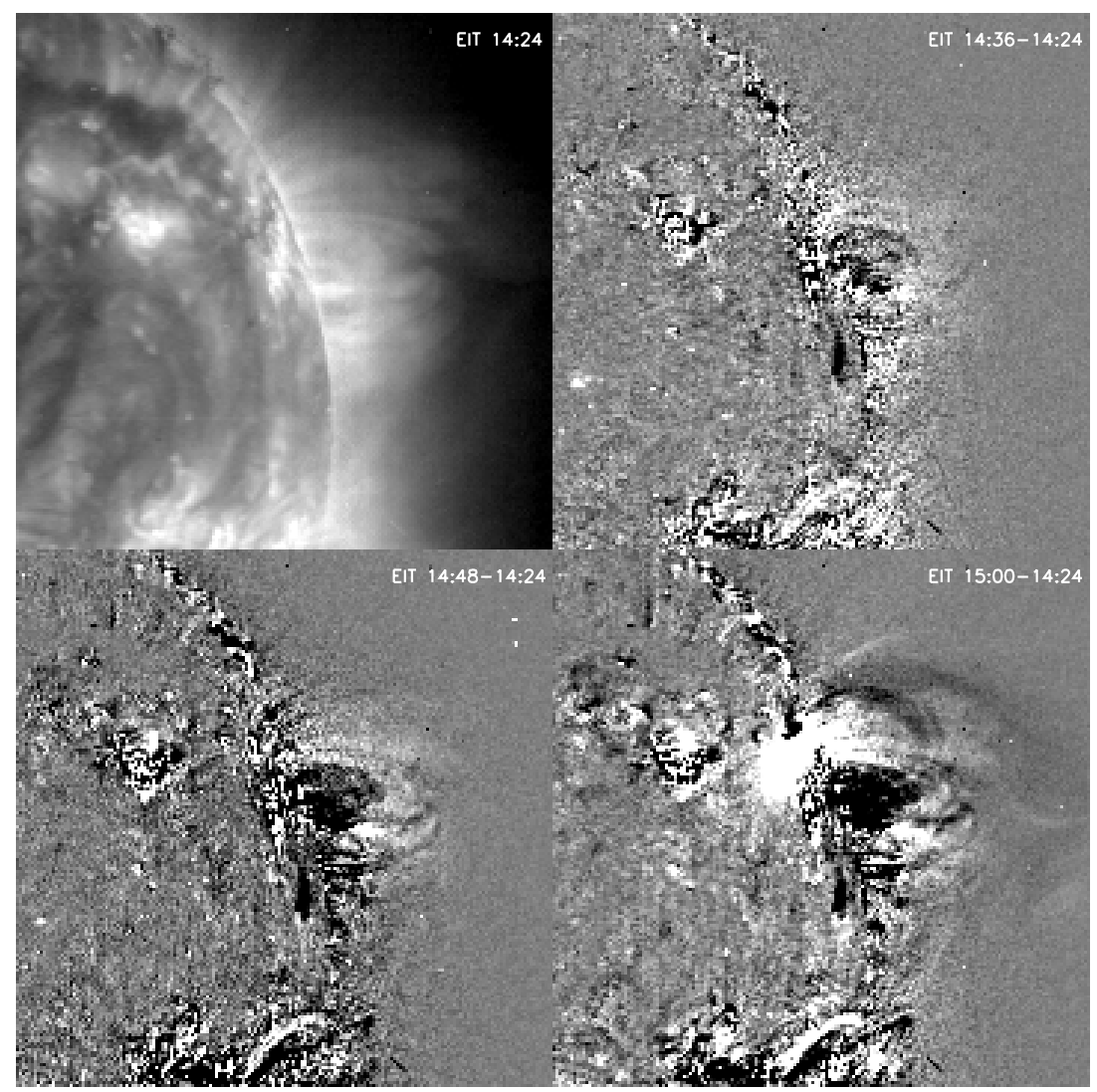

Fig. 11. EIT $195 \AA$ A data of the 2000 May 2 event. The top-left panel shows a pre-event image, while the other panels show base difference images.

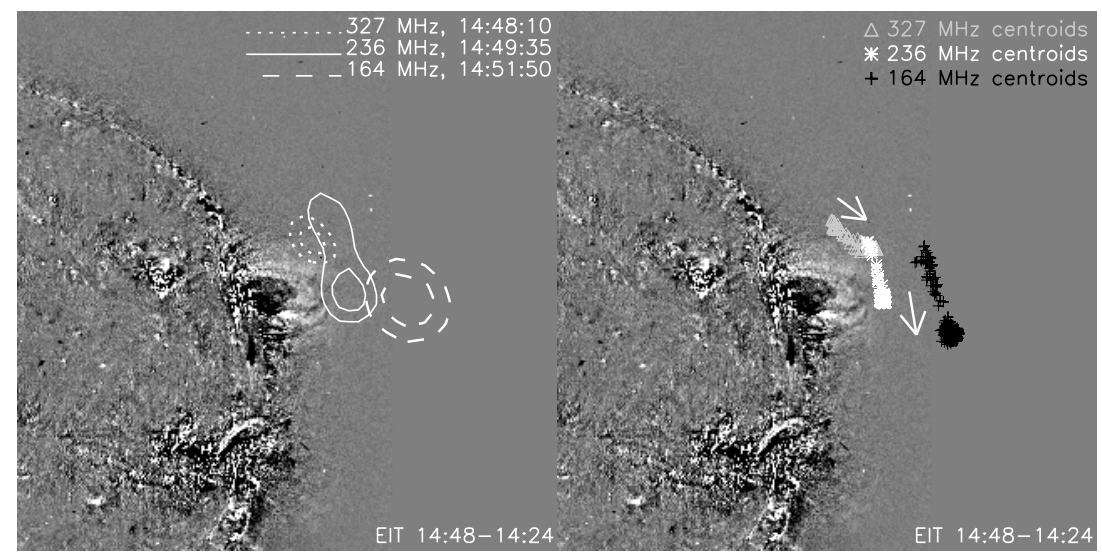

Fig. 12. The left panel shows contour plots of the 2000 May 2 type II source at 327, 236, and $164 \mathrm{MHz}$ obtained by the NRH at 14:48:10, 14:49:35, and 14:51:50 UT, respectively (contour levels at 65\% and 85\% of each frequency's maximum). The right panel shows the centroids of all NRH sources associated with the type II burst (gray triangles for $327 \mathrm{MHz}$, white stars for $236 \mathrm{MHz}$, and black crosses for $164 \mathrm{MHz}$ ). The background image is an EIT difference image. The upper arrow denotes the overall direction of motion of the type II source at $327 \mathrm{MHz}$ and the lower arrow denotes the overall direction of motion of the type II source at 236 and $164 \mathrm{MHz}$.

\section{Discussion and conclusions}

We have studied three events associated with metric type II bursts. The purpose of our study was to determine the origins of the shocks. To take advantage as much as possible of projection effects, we selected events that occurred away from disk center (longitudes equal or larger than $60^{\circ}$ ). The ambiguity about the location of the shock radio sources was partially removed by analyzing events for which the type II emission was imaged at least at two frequencies by the NRH.

In all events, both flares and CMEs were observed and their contributions to the initiation of the shocks were discussed in Sect. 3. The onset times of all type II bursts observed by ARTEMIS were estimated from the backward extrapolation of their emission lanes and were found to lie between the flare onset and the flare peak. However, this is a weak argument in favor of the flare-ignited-shock scenario in the cases where there is a tight temporal relationship between the flare and the CME as can be clearly seen for the 2000 May 2 event. We excluded the CMEs as primary drivers of the high frequency shock of 2000 March 2 and of the 2000 March 7 shock using additional arguments.

The high frequency 2000 March 2 shock is one of the fastest metric type II bursts reported in the literature. The NRH sources 


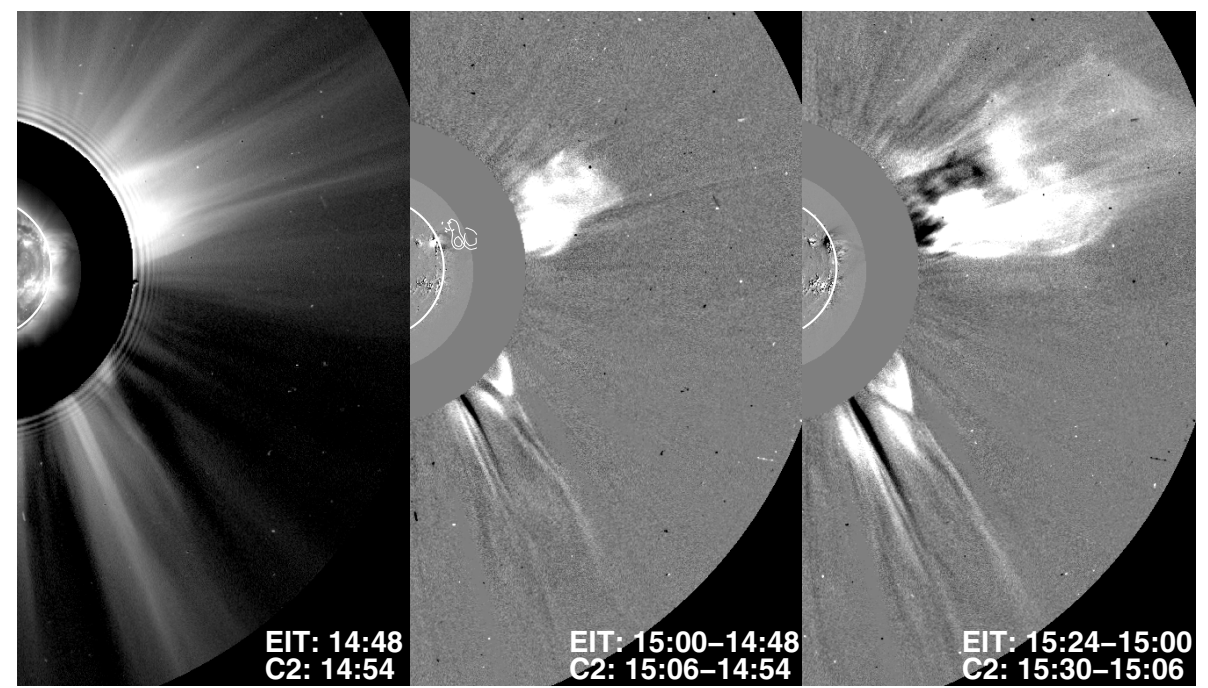

Fig. 13. Same as in Fig. 4 for the 2000 May 2 event. In the middle panel, the contours are the same as the ones in Fig. 12.

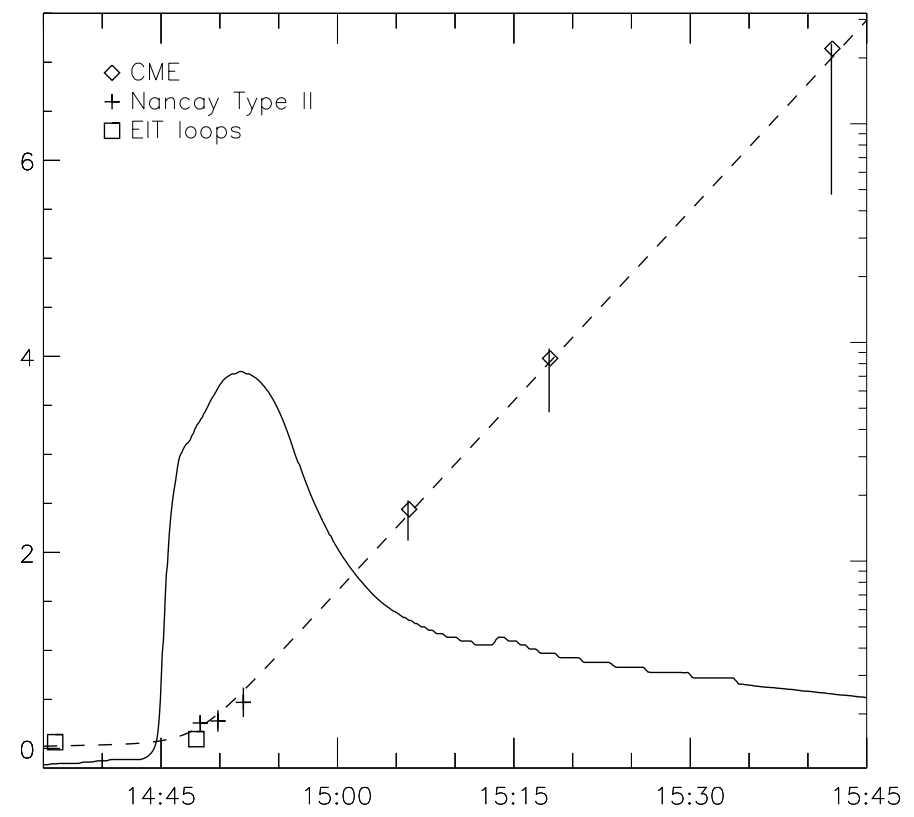

Fig. 14. Height-time profiles of various components of the 2000 May 2 event in the same format as in Fig. 5, with the following exceptions. The squares mark the heights of the expanding loops seen in EIT images and the dashed line marks the best fit of the CME heights by the function given in Eq. (2) (see text for details).

at 236 and $164 \mathrm{MHz}$ were aligned radially with respect to the flare site and allowed us to derive a speed of about $4200 \mathrm{~km} \mathrm{~s}^{-1}$ without using the drift rate or any coronal density model. On the other hand, the speed of the CME that was first observed $12.5 \mathrm{~min}$ after the end of the type II burst was about $850 \mathrm{~km} \mathrm{~s}^{-1}$. The backward extrapolation of the CME showed that it was probably related to a smaller flare that occurred prior to the one linked to the metric type II onset. The CME was observed by the LASCO C2 and C3 coronagraphs and its early evolution was missed. However, it is unlikely that it attained a speed of about $4000 \mathrm{~km} \mathrm{~s}^{-1}$ during its acceleration phase because when the acceleration phase ceases, CMEs propagate with a more or less constant speed that is similar to the speed they had acquired by the end of their acceleration (e.g. Zhang et al. 2001).

That two type II bursts were observed in close time succession during the 2000 March 2 event (i.e. the one observed by ARTEMIS and NRH and the one observed by DAM and WAVES) provides an extra argument that both of them could not have been ignited by CMEs because the LASCO observations did not show evidence of two different successive CMEs. Cases of successive type II bursts had been reported previously (e.g. Shanmugaraju et al. 2005; Subramanian \& Ebenezer 2006; Magdalenić et al. 2008). In the absence of observations of successsive CMEs, the initiation of these shocks is interpreted in terms of successive bursts of energy release in the flare or in terms of a CME driver for one of them and an ignition by the flare energy release for the other.

The origin of the low frequency type II burst of the 2000 March 2 event remains unclear. No imaging observations were available at decametric frequencies and thus its trajectory was estimated from its drift rate and the best-fit coronal density model derived from the positions of the sources of the high frequency type II burst. The resulting height of the shock radio source was $0.35 R_{\odot}$ behind the innermost feature of the CME front when the CME first appeared in the $\mathrm{C} 2$ field of view. However, this is not a strong indication of a blast wave origin of the shock because the deflection of a streamer south of the CME was observed. The streamer deflection might have been caused by a shock running ahead of the CME (e.g. Liu et al. 2009, for a recent example). If that were the case, the propagation of the shock would not be radial and the speed calculated from its drift rate would provide a lower limit to its true speed.

The 2000 March 7 type II burst was the second shock of our study for which a CME driver seemed inappropriate. The shock speed was about $2300 \mathrm{~km} \mathrm{~s}^{-1}$ and was estimated from the positions of the NRH sources at both 236 and $164 \mathrm{MHz}$. The CMEs observed by the LASCO before and after the event were not related to the shock; from the available LASCO data, their onset times were estimated 2.15 and $1.4 \mathrm{~h}$ before the appearance of the type II burst. The rise of the CMEs was not synchronized with the rise phase of the flare, while the onset time of the type II burst fell between the onset and the peak energy release of the flare. Similar cases were reported by Magdalenić et al. $(2008,2010)$.

The 2000 May 2 type II burst was imaged at three frequencies by the NRH. The trajectory of the shock was complicated: the centroids of the 236 and $164 \mathrm{MHz}$ sources were aligned radially with respect to the flare site but the $327 \mathrm{MHz}$ sources were displaced laterally. Possible interpretations were discussed in Sect. 3.2 (the sources of the 2000 March 7 type II burst also 
showed non-radial alignment with respect to the flare site). The EIT images showed expanding loops that were probably the early signatures of the CME. The acceleration phase of the CME occurred before its appearance in the $\mathrm{C} 2$ field of view. However, we were able to reconstruct it by fitting the heights of the EIT expanding loops and the heights of the white-light CME front to the same function. The acceleration peaked around the time of the appearance of the type II burst, at a height of $0.2 R_{\odot}$ and lasted $215 \mathrm{~s}$. Previous studies have shown that the ejected plasma can be accelerated within a couple of minutes and at heights as low as $0.05 R_{\odot}$ (e.g. Dere et al. 1999; Zhang et al. 2001; Gallagher et al. 2003; Williams et al. 2005). The kinematics of the CME were broadly consistent with the kinematics of the shock sources implying that the CME is the most likely cause for the shock ignition.

That two of the type II bursts we studied were not driven by CMEs does not necessarily imply that there was no material driver at all; it is possible that an initial driver (which does not develop into a large-scale CME) of coronal shocks is at work even if they later become blast waves. Unfortunately, the cadence of our EUV data was insufficient to test whether this was the case for the small-scale ejecta observed in the 2000 March 2 event and the dark feature that was activated during the 2000 March 7 event.

Our analysis illustrates the diversity of the physical conditions that may lead to the generation of coronal shocks:

1. The high speed coronal shock wave (2000 March 2) was associated with an M6.5-class flare. A CME that was observed after the end of the metric type II burst had a speed of a factor of 4-5.25 smaller than the speed of the shock. Hence, the shock could not have been ignited by the CME. That CME may have contributed to the ignition of a decametric shock that appeared just after the high speed metric shock and was related to an earlier C5.5-class flare.

2. The shock wave observed on 2000 March 7 was synchronized with the rise phase of the flare but not with the CMEs that appeared in the coronagraph data.

3. The last event (2000 May 2) included a shock wave whose kinematics were broadly consistent with the kinematics of the associated CME inferred from EUV and coronagraph data. Thus, the CME is the primary candidate for the ignition of the shock.

Acknowledgements. We thank A. Kerdraon and K.-L. Klein for providing the $\mathrm{NRH}$ visibities and the DAM data, respectively. We also thank S. Patsourakos for useful discussions.

\section{References}

Aurass, H., Hofmann, A., \& Urbarz, H.-W. 1998, A\&A, 334, 289 Cane, H. V., \& Erickson, W. C. 2005, ApJ, 623, 1180 Caroubalos, C., Maroulis, D., Patavalis, N., et al. 2001, Exp. Astron., 11, 23

Caroubalos, C., Hillaris, A., Bouratzis, C., et al. 2004, A\&A, 413, 1125

Cho, K. S., Moon, Y. J., Dryer, M., et al. 2005, JGR, 110, A1201

Cho, K. S., Lee, J., Moon, Y. J., et al. 2007, A\&A, 461, 1121

Ciaravella, A., Raymond, J. C., Kahler, S. W., Vourlidas, A., \& Li, J. 2005, ApJ, 621,1121

Classen, H. T., \& Aurass, H. 2002, A\&A, 384, 1098

Dauphin, C., Vilmer, N., \& Krucker, S. 2006, A\&A, 455, 339

Dere, K. P., Brueckner, G. E., Howard, R. A., Michels, D. J., \& Delaboudinière,

J. P. 1999, ApJ, 516, 465

Gallagher, P. T., Lawrence, G. R., \& Dennis, B. R. 2003, ApJ, 588, L53

Gary, D. E., Dulk, G. A., House, L., et al. 1984, A\&A, 134, 222
Gergely, T. E., Kundu, M. R., \& Hildner, E. 1983, ApJ, 268, 403

Gopalswamy, N., Kaiser, M. L., Lepping, R. P., et al. 1998, JGR, 103, 307

Gopalswamy, N., Yashiro, S., Kaiser, M. L., Howard, R. A., \& Bougeret, J.-L. 2001, JGR, 106, 29219

Gopalswamy, N., Aguilar-Rodriguez, E., Yashiro, S., et al. 2005, JGR, 110, A12S07

Gopalswamy, N. 2006, Amer. Geophys. Union Geophys. Monograph Ser., 165, 207

Gopalswamy, N., Thompson, W. T., Davila, J. M., et al. 2009, Sol. Phys., 259, 227

Harvey, G. A. 1965, JGR, 70, 2961

Hudson, H. S., \& Warmuth, A. 2004, ApJ, 614, L85

Kerdraon, A., \& Delouis, J.-M. 1997, in Coronal Physics from Radio and Space Observations, ed. G. Trottet, Lect. Notes Phys., 483, 192

Klassen, A., Aurass, H., Klein, K.-L., Hofmann, A., \& Mann, G. 1999, A\&A, 343, 287

Klassen, A., Pohjolainen, S., \& Klein, K.-L. 2003, Sol. Phys., 218, 197

Klein, K.-L., Khan, J. I., Vilmer, N., Delouis, J.-M., \& Aurass, H. 1999, A\&A, 346, L53

Liu, Y., Luhmann, J. G., Bale, S. D., \& Lin, R. P. 2009, ApJ, 691, L151

Magdalenić, J., Vršnak, B., Pohjolainen, S., et al. 2008, Sol. Phys., 253, 305

Magdalenić, J., Marqué, C., Zhukhov, A. N., Vršnak, B., \& Žic, T. 2010, ApJ, 718,266

Maia, D., Pick, M., Vourlidas, A., \& Howard, R. 2000, ApJ, 528, L49

Mancuso, S., Raymond, J. C., Kohl, J., et al. 2002, A\&A, 383, 267

Mann, G., Classen, H. T., \& Aurass, H. 1995, A\&A, 295, 775

Mann, G., Klassen, A., Classen, H. T., et al. 1996, A\&AS, 119, 489

Maxwell, A., \& Thompson, A. R. 1962, ApJ, 135, 138

Nelson, G. J., \& Melrose, D. B. 1985, Solar Radiophysics, ed. D. J. McLean, \& N. Labrum (Cambridge University Press), 333

Nelson, G. J., \& Robinson, R. D. 1975, Proc. Astr. Soc. Austr., 2, 370

Nindos, A., Aurass, H., Klein, K.-L., \& Trottet, G. 2008, Sol. Phys., 253, 3

Ontiveros, V., \& Vourlidas, A. 2009, ApJ, 693, 267

Patsourakos, S., Vourlidas, A., \& Kliem, B. 2010, A\&A, 522, A100

Pick, M., \& Vilmer, N. 2008, A\&AR, 16, 1

Pohjolainen, S., \& Lehtinen, N. J. 2006, A\&A, 449, 359

Pohjolainen, S., van Driel-Gesztelyi, L., Culhane, J. L., Manoharan, P. K., \& Elliott, H. A. 2007, Sol. Phys., 244, 167

Pohjolainen, S., Pomoell, J., \& Vainio, R. 2008, A\&A, 490, 357

Pothitakis, G., Preka-Papadema, P., Moussas, X., et al. 2009, in IAU Symp. 257, ed. N. Gopalswamy, \& D. F. Webb, 299

Priest, E. R. 1982, Solar Magnetohydrodynamics (Dordrecht: Reidel)

Raymond, J. C., Thompson, B. J., St. Cyr, O. C., et al. 2000, Geophys. Res. Lett., 27,1439

Reiner, M. J., Krucker, S., Gary, D. E., et al. 2007, ApJ, 657, 1107

Shanmugaraju, A., Moon, Y.-J., Cho, K.-S., et al. 2005, Sol. Phys., 232, 87

Shanmugaraju, A., Moon, Y.-J., Cho, K.-S., Dryer, M., \& Umapathy, S. 2006, Sol. Phys., 233, 117

Sheeley, N. R., Jr., Hakala, W. N., \& Wang, Y.-M. 2000, JGR, 105, 5081

Sheeley, N. R., Jr., Warren, H. P., \& Wang, Y.-M. 2007, ApJ, 671, 926

Smerd, S. F., Sheridan, K. V., \& Stewart, R. T. 1975, ApL, 16, 23

Steinolfson, R. S. 1984, Sol. Phys., 94, 193

Subramanian, K. R., \& Ebenezer, E. 2006, A\&A, 451, 683

Temmer, M., Veronig, A. M., Vršnak, B., et al. 2008, ApJ, 673, L95

Uchida, Y., Altschuler, M. D., \& Newkirk, G., Jr. 1973, Sol. Phys., 28, 495

Vourlidas, A., Wu, S. T., Wang, A. H., Subramanian, P., \& Howard, R. A. 2003, ApJ, 598, 1392

Vršnak, B. 2001a, JGR, 106, 25291

Vršnak, B. 2001b, Sol. Phys., 202, 173

Vršnak, B., \& Cliver, E. W. 2008, Sol. Phys., 253, 215

Vršnak, B., Ruždjak, V., Zlobec, P., \& Aurass, H. 1995, Sol. Phys., 158, 331

Vršnak, B., Aurass, H., Magdalenić, J., \& Gopalswamy, N. 2001, A\&A, 377, 321

Vršnak, B., Magdalenić, J., Aurass, H., \& Mann, G. 2002, A\&A, 396, 673

Vršnak, B., Warmuth, A., Temmer, M., et al. 2006, A\&A, 448, 739

Warmuth, A., Vršnak, B., Magdalenić, J., Hanslmeier, A., \& Otruba, W. 2004, A\&A, 418, 1117

White, S. M., Mercier, C., Bastian, T., \& Bradley, R. 2007, NRAO Newsletter, 110,9

Williams, D. R., Török, T., Démoulin, P., van Driel-Gesztelyi, \& Kliem, B. 2005, ApJ, 628, L163

Zhang, J., Dere, K. P., Howard, R. A., Kundu, M. R., \& White, S. M. 2001, ApJ, 559,452

Zhang, J., Dere, K. P., Howard, R. A., \& Vourlidas, A. 2004, ApJ, 604, 420 